Trauma Berufskrankh 2016 • 18 (Suppl 5):S430-S433 DOI 10.1007/s10039-016-0149-2

Online publiziert: 2. Mai 2016

(c) Springer-Verlag Berlin Heidelberg 2016

CrossMark

\title{
Matthias Aurich
}

Sana Klinikum Borna, Klinik für Orthopädie und Unfallchirurgie, Sana Kliniken Leipziger Land, Borna, Deutschland

\section{Korrekturen durch Gelenkersatz}

\section{Posttraumatische Kniegelenkendoprothetik}

Frakturen des Kniegelenkes, Bandverletzungen, Meniskusresektionen und isolierte Knorpeldefekte sind als Mitursache einer posttraumatischen Gonarthrose anzusehen. Folgende Faktoren beeinflussen dabei die Entwicklung eines Gelenkverschleißes:

- erhöhte Druckbelastung/Verkleinerung der Kontaktfläche,

- Instabilitäten/unphysiologische Scherbelastung,

- Fehlbelastungen/Achsenabweichungen.

Bis $\mathrm{zu} 45 \%$ der Patienten entwickeln nach Frakturen am Kniegelenk arthrotische Veränderungen [6]. Kniegelenknahe Frakturen stehen demnach im Mittelpunkt des Interesses. Die prinzipiellen Behandlungsmöglichkeiten sind:

- konservativ,

- Rekonstruktion durch Osteosynthese,

- Gelenkersatz durch eine Endoprothese.
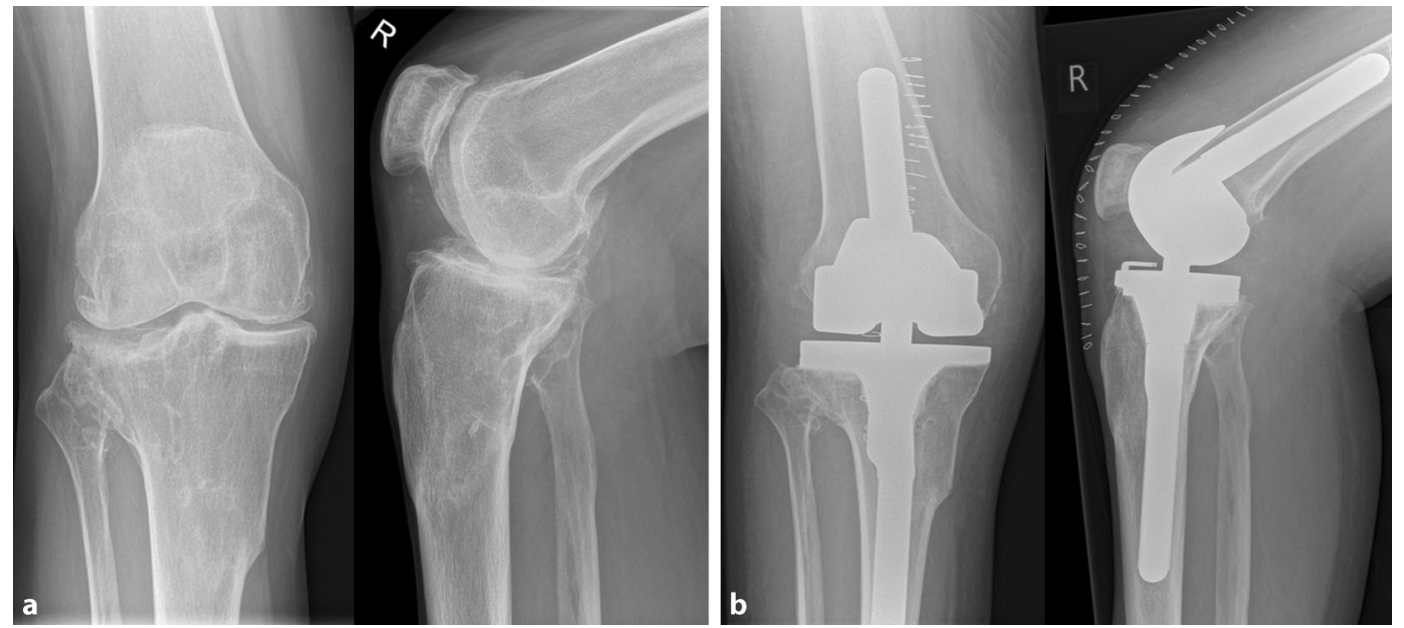

Abb. $1<$ a Röntgenbilder in 2 Ebenen der posttraumatischen Gonarthrose einer 72-jährigen Patientin nach Tibiakopffraktur vor 13 Jahren. b Die Versorgung erfolgte mit einer gekoppelten modularen Knietotalendoprothese aufgrund der instabilen Valgusfehlstellung 
Anstieg von kniegelenknahen Frakturen im Alter zu verzeichnen [10].

Bei Vorliegen folgender Faktoren sollte der primäre Gelenkersatz geprüft werden:

- schlechte Knochenqualität,

- keine Entlastung/Teilbelastung möglich,

- vorbestehende Gonarthrose,

- multiple Begleiterkrankungen.

Die Indikationen für einen sekundären Gelenkersatz nach kniegelenknahen Frakturen sind [4]:

- posttraumatische Gonarthrose,

- in Fehlstellung verheilte Fraktur,

- komplexe Bandinstabilitäten.

Dabei ist zu beachten, dass die Komplikationsrate im Vergleich zur Totalendoprothese (TEP) bei primärer Gonarthrose erhöht ist $[12,18]$.

Eine wichtige Frage ist also, wann der richtige Zeitpunkt des Gelenkersatzes nach einer Fraktur gekommen ist.

Verschiedene Literaturangaben zeigen eine bis zu 15 \%ige Häufigkeit einer sekundären Knie-TEP-Versorgung nach primär osteosynthetisch versorgter Tibiakopffraktur [15, 18, 21].

Wasserstein et al. [20] untersuchten 8426 operativ versorgte Tibiakopffrakturen (Gruppe 1) hinsichtlich der Notwendigkeit einer sekundären Knie-TEPVersorgung und verglichen dies mit der Normalbevölkerung ohne Fraktur ( $N=$ 33.698, Gruppe 2) im selben Zeitraum. Dabei zeigte sich in der Gruppe 1 eine Häufigkeit von 5,3\% nach 5 Jahren bzw. $7,3 \%$ nach 10 Jahren, im Vergleich zu $0,82 \%$ bzw. $1,8 \%$ in Gruppe 2. Die Autoren schlussfolgern, dass eine operativ versorgte Tibiakopffraktur die Wahrscheinlichkeit für die Implantation einer KnieTEP deutlich erhöht und dass dies zudem mit höherem Patientenalter, einer bikondylären Fraktur und höherer Komorbidität assoziiert ist.

Weiss et al. [22] untersuchten 13.821 primäre Knie-TEPs und konnten dabei lediglich bei 110 Patienten $(0,8 \%)$ eine kniegelenknahe Frakturin der Anamnese feststellen. Die Autoren schlussfolgern, dass die Notwendigkeit einer sekundären Knie-TEP nach kniegelenknaher Fraktur mittelfristig eher gering ist. Daher be-

Trauma Berufskrankh 2016 · 18 (Suppl 5):S430-S433 DOI 10.1007/s10039-016-0149-2

(c) Springer-Verlag Berlin Heidelberg 2016

\section{Aurich}

\section{Korrekturen durch Gelenkersatz. Posttraumatische Kniegelenkendoprothetik}

\section{Zusammenfassung}

Die posttraumatische Arthrose (PTA) des Kniegelenkes ist eine häufige Komplikation nach intra- und extraartikulären Frakturen. Darüber hinaus kann die PTA aber auch Folge von isolierten Knorpeldefekten, Meniskusresektionen und Bandverletzungen sein. Es gibt verschiedene Behandlungsmethoden von kniegelenknahen Frakturen. In Endstadium einer PTA jedoch ist bei ausgereizter konservativer Behandlung der endoprothetische Gelenkersatz die Methode der Wahl. Die primäre Endoprothese nach kniegelenknaher Fraktur ist momentan auch an großen Zentren ein seltener Eingriff. Sie ist indiziert bei älteren Patienten mit der Unfähigkeit zur Entlastung, bei einer erheblichen knöchernen Destruktion, in Fällen mit einer vorbestehenden symptomatischen Gonarthrose beim älteren Patienten, und ist oft mit dem primären Einsatz eines achsgeführten und modularen Implantates verbunden. Die Indikation zur primären Kniegelenktotalendoprothese nach Fraktur ist im klinischen Alltag eher eine Einzelfallentscheidung. Die sekundäre Endoprothetik nach kniegelenknaher Fraktur zeigt prinzipiell gute funktionelle Ergebnisse, wobei die Resultate der Arthroplastik nach primärer Arthrose nicht zu erreichen sind. Zudem sind die Komplikationsraten wesentlich höher. Häufig sind Probleme mit dem Streckapparat bei Status nach Tibiakopffrakturen. Darüber hinaus können Probleme aufgrund von bestehenden Narben, einer Pseudarthrose (ggf. auf dem Boden einer möglichen Low-grade-Infektion), Malalignement, Bewegungseinschränkungen oder Instabilität auftreten. Daher ist die Implantation einer Kniegelenktotalendoprothese bei PTA deutlich aufwendiger als bei primärer Gonarthrose.

\section{Schlüsselwörter}

Gonarthrose · Fraktur · Komplikation · Arthrose - Endoprothese

\section{Correction by joint replacement. Posttraumatic knee joint prosthetics}

\section{Abstract}

Posttraumatic arthritis (PTA) of the knee is a common complication after intraarticular and extra-articular fractures; moreover, PTA may also be a result of isolated cartilage defects, meniscus resection and ligament injuries. There are various methods of treatment for knee joint fractures; however, in the final stage of PTA, when conservative treatment fails endoprosthetic joint replacement is the method of choice. Primary total knee replacement (TKR) for the treatment of fractures close to the knee joint is currently a rare intervention even at major centers. It is indicated in elderly patients with no possibility of relief, in the case of substantial bone destruction, in cases with presymptomatic osteoarthritis in the elderly and is often associated with the primary use of a modular implant. The indications for primary TKR after knee joint fractures should always be made on an individual basis in the clinical routine. Secondary TKR after fractures close to the knee joint generally shows good functional results, although inferior when compared with the results of TKR for primary osteoarthritis (OA). In addition, the complication rates of TKR for PTA are much higher. Problems with the extensor mechanism after tibial plateau fractures are common. In addition, there are problems caused by pre-existing scars, nonunion (possibly due to a low grade infection), malalignment, restricted movement and instability; therefore, implantation of a TKR in PTA is much more difficult than in primary OA.

\section{Keywords}

Knee osteoarthritis · Fracture - Joint replacement $\cdot$ Complication $\cdot$ Arthritis 
steht aus deren Sicht keine allgemeine Indikation zur primären Knie-TEP nach kniegelenknahen Frakturen.

Die primäre Versorgung einer Tibiakopffraktur mit einer Knie-TEP zeigt Literaturangaben zufolge in lediglich $55-65 \%$ sehr gute mittelfristige Ergebnisse [19].

Eine primäre Knie-TEP nach distaler Femurfraktur ist deutlich seltener, und es gibt nur einige wenige Fallstudien [1, 2, 14]. Meist sind ältere, multimorbide Patienten betroffen, und es werden oft komplexe Implantate, wie z. B. ein distaler Femurersatz, benötigt.

Probleme bei der Primärversorgung sind insbesondere die knöcherne Defektsituation sowie Instabilitäten. Daher ist eine Schlittenversorgung überhaupt nicht, ein bikondylärer Oberflächenersatz nur selten möglich. Zumeist erfolgt die Implantation mit achsgeführten Prothesen in zementierter Technik bzw. die Verwendung von modularen RevisionsTEPs [8, 10].

Als Indikationen für einen primären Gelenkersatz nach kniegelenknaher Fraktur werden gesehen $[5,8-10,16]$ :

- fortgeschrittene symptomatische

Gonarthrose beim älteren Patienten,

- primäre Vollbelastung erforderlich

(ältere Patienten ohne Teilbelastungsfähigkeit),

- erhebliche Zerstörung der Gelenkfläche mit nichtrekonstruierbarem

Defekt bei älteren Patienten,

- begleitende komplexe Instabilität beim älteren Patienten.

Als Kontraindikationen gelten:

- Infektsituation am verletzten Knie,

- schwere Weichteilverletzungen,

- nichtrekonstruierbarer Streckapparat,

- schweres neurologisches Defizit (z. B. Femoralisläsion),

- metaphysärer Substanzverlust (Trümmerzone),

- junger Patient,

- fehlende Compliance.

Eine Übersichtsarbeit von König et al. [11] schlussfolgerte daher: „Die Indikation zur primären Knie-TEP nach Fraktur ist im klinischen Alltag eher eine Einzel- fallentscheidung.“ Dies ist in jedem Fall zu unterstützen.

Die Indikationen einer sekundären Knie-TEP nach kniegelenknaher Fraktur sind ähnlich wie bei einer primären Gonarthrose, d.h. bei Vorliegen von Ruheschmerz, Belastungsschmerz und Funktionsverlust des Kniegelenkes, sowie infolge eines Osteosyntheseversagens nach kniegelenknaher Fraktur. Hierbei ist insbesondere ein Infekt (Low-gradeInfekt) auszuschließen.

Die Abb. 1 zeigt den Fall einer 72jährigen Patientin mit posttraumatischer Gonarthrose nach Tibiakopffraktur vor 13 Jahren. Bei entsprechender Beschwerdesymptomatik wurde die Indikation zum Gelenkersatz gestellt. Aufgrund der ausgeprägten Valgusfehlstellung mit massiver Seitenbandinstabilität erfolgte die Versorgung mit einer gekoppelten Endoprothese.

$\mathrm{Zu}$ beachten sind die unterschiedlichen Ergebnisse in der Literatur sowie die höhere Komplikationsrate im Vergleich zur Knie-TEP bei primärer Gonarthrose [13].

Gerich et al. [3] berichten über Kniegelenkendoprothetik nach Tibiakopffrakturen und vergleichen dabei die KnieTEP bei primärer Gonarthrose (Gruppe 1) mit der sekundären Knie-TEP nach kniegelenknaher Fraktur (Gruppe 2). Hierbei zeigen sich in Gruppe 2 eine postoperativ höhere Schmerzstärke (visuelle Analogskala [VAS]), eine postoperativ schlechtere Alltagsfunktion, eine erhöhte Revisionsrate (27,3 vs. $10,5 \%$ ) sowie Probleme im Streckapparat bei Zustand nach Tibiakopffraktur.

Scott et al. [17] konnten in ihrer Studie keine Unterschiede in den klinischen Scores feststellen. Es zeigten sich jedoch ebenfalls gehäuft Probleme mit dem Streckapparat bei Zustand nach Tibiakopffrakturen.

In der Studie von Weiss et al. [22] zeigten sich sehr gute Ergebnisse in $78 \%$ nach Tibiakopffrakturen, aber nur in $52 \%$ nach distaler Femurfraktur. Als Komplikationen wurden Infekte, Weichteilprobleme und Probleme mit dem Streckapparat bei Zustand nach Tibiakopffrakturen angegeben.

Die sekundäre Knie-TEP nach kniegelenknahen Frakturen zeigt zusammen- fassend relativ gute funktionelle Ergebnisse bei jedoch höherer Komplikationsrate im Vergleich zur Knie-TEP bei primärer Gonarthrose. Die besten Ergebnisse werden nach isolierten Tibiakopffrakturen erreicht. Problematisch sind kombinierte Frakturen von Tibia und Femur sowie vorhandene Weichteilschäden. $\mathrm{Zu}$ beachten sind zudem vermehrt Probleme mit dem Streckapparat bei Zustand nach Tibiakopffraktur.

\section{Fazit für die Praxis}

- Die primäre Endoprothese nach kniegelenknaher Fraktur ist momentan auch an großen Zentren ein seltener Eingriff. Sie ist indiziert bei älteren Patienten mit der Unfähigkeit zur Entlastung, bei einer erheblichen knöchernen Destruktion in Fällen mit einer vorbestehenden symptomatischen Gonarthrose beim älteren Patienten, und sie ist oft mit dem primären Einsatz eines achsgeführten und modularen Implantates verbunden. Die Indikation zur primären Knie-TEP nach Fraktur ist im klinischen Alltag eher eine Einzelfallentscheidung.

- Die sekundäre Endoprothetik nach kniegelenknaher Fraktur zeigt prinzipiell gute funktionelle Ergebnisse, wobei die Resultate der Arthroplastik nach primärer Arthrose nicht zu erreichen sind. Zudem sind die Komplikationsraten wesentlich höher. Häufig sind Probleme mit dem Streckapparat bei Status nach Tibiakopffrakturen.

\section{Korrespondenzadresse}

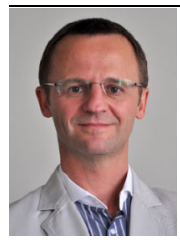

PD Dr. med. habil. M. Aurich

Sana Klinikum Borna, Klinik für Orthopädie und Unfallchirurgie, Sana Kliniken Leipziger Land Rudolf-Virchow-Str. 2 04552 Borna, Deutschland matthias.aurich@sana.de 


\section{Einhaltung ethischer Richtlinien}

Interessenkonflikt. M. Aurich gibt an, dass kein Interessenkonflikt besteht.

Dieser Beitrag beinhaltet keine vom Autor durchgeführten Studien an Menschen oder Tieren.

The supplement containing this article is not sponsored by industry.

\section{Literatur}

1. Appleton P, Moran M, Houshian S, Robinson CM (2006) Distal femoral fractures treated by hinged total knee replacement in elderly patients. J Bone Joint Surg Br 88-B:1065-1070

2. Fakler JKM, Hepp P, Marquaß B, Dercks N von, Josten C (2013) Ist der distale Femurersatz als extremitätenerhaltendes Verfahren nach komplexenFrakturen am distalen Femurgeeignet? ZOrthop Unfall 151:173-179

3. Gerich T, Bosch U, Schmidt E, Lobenhofer P, Krettek C (2001) Kniegelenksendoprothetik nach Tibiakopffrakturen. Unfallchirurg 104:414-419

4. Großstück R, Conrad T, Deml O, Brodt S, Hofmann GO (2008) Standards in der Endoprothetik nach Gelenkverletzungen. Trauma Berufskrankh 10:155-170

5. Hofmann GO (2013) Endoprothetik bei Kniegelenksfrakturen. Trauma Berufskrankh 13(Suppl.1):130-132

6. Honkonen SE (1995) Degenerativ arthritis after tibia plateau fractures. J Orthop Trauma 9:273-277

7. Jensen DB, Rude C, Duus B, Bjerg-Nielsen A (1990) Tibial plateau fractures. A comparison of conservative and surgical treatment. J Bone Joint Surg Br 72(1):49-52

8. Kilian U (2003) Kniegelenksendoprothese zur primären Therapie der intraartikulären Tibiakopffraktur im höheren Alter. Unfallchirurg 106:1046-1050

9. KiniSG, SathappanSS (2013) Role of navigated total knee arthroplasty for acute tibial fractures in the elderly. Arch Orthop Trauma Surg 133:1149-1153

10. Klösters C, Schliemann B, Raschke MJ (2011) Tibiakopffrakturen im Alter. Unfallchirurg 114:251-262

11. König V, Dumpies CW, Hoffmann GO (2015) Frakturendoprothetik am Kniegelenk: Wann primär? Wann sekundär? Trauma Berufskrankh 17:182-188

12. Lizaur-Utrilla A, Collados-Maestre I, MirallesMunoz FA, Lopez-Prats FA (2015) Total knee arthroplasty for osteoarthritis secondary to fracture of the tibial plateau. A prospektive matched cohort study. J Arthroplasty 30(8):1328-1332. doi:10.1016/j.arth.2015.02.032

13. MarczakDM, Synder M, Sibinski M, Okon T, Kowalczewski J (2014) One-stage total knee arthroplasty with pre-existing fracture deformity post-fracture total knee arthroplasty. J Arthroplasty 29:2104-2108

14. Mounasamy V, Cui Q, Brown TE, Saleh KJ, Mihalko WM (2007) Primary total knee arthroplasty for a complex distal femur fracture in the elderly: a case report. Eur J Orthop Surg Traumatol 17:491-494

15. Rademakers MV, Kerkhoffs GMMJ, Sierevelt IN Rademakers ELFB, Marti RK (2007) Operative treatment of 109 tibial plateau fractures: fiveto 27-year follow-up results. J Orthop Trauma 21(1):5-10
16. Schwarz N, Buchinger W, Mähring M, Schedl R, Wildhalm HK (2008) Knieendoprothese als Ersttherapie bei proximaler Tibiafraktur. Unfallchirurg 111:928-932

17. Scott CEH, Davidson E, Mac Donald DJ, White TO, Keating JF (2015) Total knee arthroplasty following tibial plateau fracture. Bone Joint J 97-B:532-538

18. Simpson D, Keating JF (2004) Outcome of tibial plateau fractures management with calcium phosphate cement. Injury 35(9):913-918

19. Vermeire J, Scheerlinck T (2010) Early primary total knee replacement for complex proximal tibia fractures in elderly and osteoarthritic patients. Acta Orthop Belg 76:785-793

20. Wasserstein D, Henry P, Paterson JM, Kreder HJ, Jenkinson R (2014) Risk of total knee arthroplasty after operatively treated tibial plateau fracture. $J$ Bone Joint Surg Am 96-A:144-150

21. Weigel DP, Marsh JL (2002) High energy fractures of the tibial plateau. Knee function after longer follow-up. J Bone Joint Surg Am 84A(9):1541-1551

22. Weiss NG, Parvizi J, Hanssen AD, Trousdale RT, Lewallen DG (2003) Total knee arthroplasty in posttraumatic arthrosis of the knee. J Arthroplasty 18(Suppl. 1):23-26 